\title{
SURVIELLANCE OF BACTERAEMIC PATTERN IN A TERTIARY LEVEL NEONATAL INTENSIVE CARE UNIT
}

\author{
P. Arora, A.E. Abdelhamid, M. Ramaswamy, S. Fang \\ Neonates, Homerton University Hospital, London, UK
}

Background: Sepsis remains one of the major challenges in neonatal practice.

Objective: To review the trend of positive blood culture in a tertiary neonatal unit.

Methods: Retrospective analysis of all positive blood culture results from 2002 to 2009.

Results: Of the 840 positive blood cultures gram-positive bacteria accounted for $82 \%$ (687), gram-negative for $14.5 \%$ (122), and fungal isolates for $3.5 \%$ (31). There were almost equal number of males and females.

Early onset infections accounted for $10 \%$ (85), with $37 \%$ (32) in 24-28 weeks gestation and $34 \%$ (29) in babies greater than 36 weeks. The commonest organism was Coagulase-negative Staphylococci (CoNS) 47 (55\%) followed by GBS $16(19 \%)$ and Escherecia coli in $5(6 \%)$.

Late-onset infections(755 positive cultures) was much more common in preterm with $75 \%$ (568) in $24-28$ weeks gestation and $2.7 \%(21)$ in more than 36 weeks. The commonest organism being CoNS 495 (65.5\%), Group D streptococcus 46 (6\%), Klebsiella 34 (4.5\%), Candida 31 (4.1\%), Escherechia coli 27 (3.5\%), Enterobacter cloacae 16 (2.1\%), Serratia 13 (1.7\%), and Pseudomonas aerogenosa $9(1.1 \%)$.

Gram-negative infections have shown an upward trend. E.coli, Klebsiella, and Pseudomonas had a mortality rate $44 \%(33 / 75)$. Fungal infections had $48.8 \%$ mortality. Males had fatal outcome in $18 \%$ as compared to females $29 \%$.

Conclusions: Gram-negative sepsis is on the rise and has a high mortality. As fungal sepsis was very likely to be fatal, prophylactic antifungal agents in extreme preterm babies are a useful adjunct. 